\title{
n-3 polyunsaturated fatty acids reverse the development of polyps in $\mathrm{Apc}^{\mathrm{Min} /+}$ transgenic mice
}

\author{
MARIA NOTARNICOLA, VALERIA TUTINO, MARIA GABRIELLA CARUSO and ANTONIO FRANCAVILLA \\ Laboratory of Nutritional Biochemistry, National Institute for Digestive Diseases, Castellana Grotte, I-70013 Bari, Italy
}

Received June 12, 2015; Accepted July 23, 2015

DOI: $10.3892 /$ or.2015.4359

\begin{abstract}
Dietary n-3 polyunsaturated fatty acids (n-3 PUFAs) have been demonstrated to reduce tumor load in $\mathrm{Apc}^{\mathrm{Min} /+}$ mice, supporting a role for n-3 PUFAs in the inhibition of colon carcinogenesis and progression. The aim of the present study was to investigate whether a diet enriched with n-3 PUFAs, known already to have anti-neoplastic efficacy in $\mathrm{Apc}^{\mathrm{Min} /+}$ mice, would reverse the development of intestinal polyps. For this purpose, $\mathrm{Apc}^{\mathrm{Min} /+}$ mice were randomly divided into 3 groups of 5 animal each and fed as follows: control ST1 and ST2 groups, received a purified AIN-93M standard diet for 5 and 10 weeks, respectively; the OM-3R group received a purified AIN-93M standard diet for 5 weeks and a diet supplemented with salmon oil, rich in n-3 PUFAs, for another 5 weeks. After dietary treatment, in intestinal tissue, we evaluated the polyp number and volume, expression levels of cell proliferation- and apoptosis-related proteins, as well as the protein expression of LDL receptor and the levels of fatty acid synthase (FAS) activity. The results showed the ability of a diet enriched with n-3 PUFAs to suppress intestinal polyps in $\mathrm{Apc}^{\mathrm{Min} /+}$ mice, and to significantly reverse polyp development associated with the downregulation of cell proliferation markers and with the induction of estrogen receptor $\beta$ and LDL receptor, which are negative modulators of cellular proliferation. This noteworthy finding is important for a translational study evaluating the therapeutic role of n-3 PUFAs in the prevention and treatment of subjects with gastrointestinal diseases.
\end{abstract}

\section{Introduction}

Epidemiological studies have indicated that consumption in the diet of fish oil, rich in n-3 polyunsaturated fatty acids (n-3 PUFAs) has been associated with a reduced risk of

Correspondence to: Dr Maria Notarnicola, Laboratory of Nutritional Biochemistry, National Institute for Digestive Diseases, 27 Turi Street, Castellana Grotte, I-70013 Bari, Italy

E-mail: maria.notarnicola@irccsdebellis.it

Key words: n-3 polyunsaturated fatty acids, colorectal cancer, intestinal polyp, estrogen receptor, LDL receptor colorectal cancer via modulation of the inflammatory status of cellular membranes (1-3). Experimental studies have identified a role for n-3 PUFAs in colon carcinogenesis showing a growth inhibitory effect on the progression of malignancy (4). Dietary n-3 PUFAs have been demonstrated to reduce tumor load in mice with an APC defect $(5,6)$. Additionally, clinical evidence showed that these fatty acids have specific effects on disease prevention, reducing rectal polyp number and size in patients with familial adenomatous polyposis (7).

Previously, in HepG2 hepatoma cells, we showed that the exposure of these cells to the eicosapentaenoic acid (EPA) and the arachidonic acid (ARA), belonging to the family of n-3 PUFAs and n-6 PUFAs, respectively, led to cell growth arrest and the promotion of apoptosis (8). The inhibition of cell growth exerted by these PUFAs was due to a strong inhibition of fatty acid synthase (FAS) and 3-hydroxy-3-methyl-glutaryl CoA reductase (HMGCoAR) gene expression.

We previously performed an in vivo study demonstrating that Mediterranean diet components, such as olive oil, n-3 and n-6 PUFAs, when given to mice that spontaneously develop intestinal polyps ( $\mathrm{Apc}^{\mathrm{Min} /+}$ mice), are able to reduce polyp number and volume by decreasing proliferation and increasing pro-apoptotic activity (9). These biological effects were associated with the inhibition of FAS and HMGCoAR gene expression and activity as well as an increased estrogen receptor $\beta /$ estrogen receptor $\alpha(E R \beta / E R \alpha)$ ratio.

Previous findings have demonstrated that ER $\beta$ is abundantly expressed in normal colon but exhibits a progressively decreased expression in human adenomatous sporadic polyps and in $\mathrm{Apc}^{\mathrm{Min} /+}$ mice (10-15). By contrast, $\mathrm{ER} \alpha$ is a well-known mediator of cell proliferation activity (16), which acts by enhancing the transcription of factors associated with cell proliferation and shows an increased expression in colon cancer as compared to normal surrounding tissue (16). In particular, ER $\alpha$ protein expression has been demonstrated to play a role in the regulation of the hedgehog $(\mathrm{Hh})$ signaling pathway which has been demonstrated to be activated in many types of cancer, including colorectal cancer (17-19). Activation of the Hh pathway induces an overexpression of Hh signaling pathway-associated genes, sonic hedgehog (Shh) protein and the glioma-associated oncogene homolog-1 (GLI-1) $(19,20)$.

Another important feature of malignant transformation is loss of the cholesterol feedback inhibition mechanism that regulates cholesterol synthesis $(21,22)$. The main cholesterol feedback defect in malignant cells has been located at the 
HMGCoAR step. Cancer cells are characterized by a reduced expression of LDL receptor (LDL-R) and as a consequence of the inability to internalize the exogenous cholesterol, tend to increase the endogenous synthesis, thereby increasing the activity of HMGCoAR. Alterations of lipid metabolism, and high levels of FAS and HMGCoAR expression and activity, are usual characteristics of tumor cells and are essential in the onset and progression of tumor (23).

The aim of the present study was to investigate whether a diet enriched with n-3 PUFAs, known already to have an anti-neoplastic efficacy, would reverse the development of intestinal polyps in $\mathrm{Apc}^{\mathrm{Min} /+}$ mice and to examine the possible molecular mechanisms involved. The expression levels of cell proliferation- and apoptosis-related proteins, as well as the protein expression of LDL-R and the levels of FAS activity were analyzed in mouse intestinal tissue.

\section{Materials and methods}

Animals and experimental study design. Five-week-old C57BL/6J male mice with a heterozygote mutation for the Apc gene $\left(\mathrm{Apc}^{\mathrm{Min} /+}\right)$ were obtained from Charles River Laboratories Italia (Calco, Italy). The mice were maintained under temperature-, air- and light-controlled conditions and received food and water ad libitum, although they did not receive any surgical or hormonal manipulation. All animals received care in compliance with the 'Guide for the Care and Use of Laboratory Animals'. The procedures regarding animal use were communicated to the Italian Ministry of Health and approved.

The $\mathrm{Apc}^{\mathrm{Min} /+}$ mice were randomly divided into 3 groups of 5 animals each and fed as follows: control (ST1) group, received a purified AIN-93M standard diet (12.5\% protein, $12 \%$ soybean oil, $3 \%$ cellulose fiber) for 5 weeks; control (ST2) group, received the same purified AIN-93M standard diet for 10 weeks; OM-3R group, received a purified AIN-93M standard diet for 5 weeks and a purified AIN-93M standard diet in which soybean oil was replaced with salmon oil, rich in n-3 PUFAs (12.5\% protein, $12 \%$ salmon oil, 3\% cellulose fiber) for an additional 5 weeks. Diet fatty acid composition has been previously described (9), with the diets being isocaloric and supplied as pellets (Mucedola Srl, Settimo Milanese, Italy). Body weight and food intake of the mice were measured every 3 days.

Following establishment of the time period for the dietary treatment, the animals were sacrificed by cervical dislocation and the entire intestinal tract was immediately removed and washed with cold phosphate-buffered saline (PBS). The small intestine and colon were cut along the mesenteric insertion, placed on a paper strip at $0-4^{\circ} \mathrm{C}$ and analyzed through a stereomicroscope at $\mathrm{x} 3$ magnification in order to calculate the number and the volume of polyps. The small intestine was further divided into proximal, medial and distal segments. A portion of the intestinal segments of all the animals was immediately stored at $-80^{\circ} \mathrm{C}$, for western blot analysis and enzymatic activity analyses.

Western blotting. Protein expression levels of ER $\alpha, \mathrm{ER} \beta$, Shh, GLI-1, STAT3 and p-STAT3Ser as well as PIAS-3, caspase-8, $\mathrm{Bax}, \mathrm{Bcl}-2$, LDL-R and $\beta$-actin protein expression were evaluated in distal tissue specimens by western blot analysis.
Briefly, $50 \mu \mathrm{g}$ aliquots of total protein were separated in 4-12\% pre-cast polyacrylamide gels (Invitrogen, Life Technologies, Carlsbad, CA, USA) and transferred onto a PVDF membrane with Transblot Turbo (both from Bio-Rad Laboratories, Milan, Italy). The primary antibodies anti-p-STAT3Ser, antiSTAT3 and anti- $\beta$-actin (Cell Signaling Technology, Beverly, MA, USA); anti-ER $\alpha$, anti-ER $\beta$, anti-Bax, anti-Bcl-2, anticaspase-8, anti-Shh, anti-GLI-1 and anti-PIAS-3 (Santa Cruz Biotechnology, Inc., Santa Cruz, CA, USA); and anti-LDL-R (Abcam, Cambridge, MA, USA) were diluted at 1:500 in blocking buffer. After overnight incubation, the membranes were subsequently incubated with a horseradish peroxidaseconjugated secondary antibody (Bio-Rad Laboratories). The proteins were detected by chemiluminescence (ECL; Thermo Scientific, Rockford, IL, USA) and the densitometric analysis of each protein-related signal was obtained using the Molecular Imager Chemidoc ${ }^{\mathrm{TM}}$ (Bio-Rad Laboratories) and normalized against $\beta$-actin expression.

FAS activityassay.FAS activity was determined on frozen distal intestinal samples, as previously described (24), and expressed as picomoles of incorporated $2-{ }^{14} \mathrm{C}$-malonyl-CoA $/ \mathrm{min} / \mathrm{mg}$ of total proteins.

Statistical analysis. Data are presented as means \pm SE. The significance of the differences among experimental groups was evaluated by one-way analysis of variance (ANOVA) and Tukey's multiple comparison test. Differences were considered significant at a $\mathrm{P}<0.05$.

\section{Results}

Total number and volume of intestinal polyps. The number and volume of polyps evaluated along the entire intestinal tract in three groups of mice were determined (Fig. 1A and B). Compared to the mice treated with standard diet for 5 weeks (ST1 group), the number of polyps in the OM-3R group was decreased although the reduction was not statistically significant. However, a statistically significant difference in polyp number was present between the ST2 group (mice treated with standard diet for 10 weeks) and the OM-3R group ( $\mathrm{P}=0.03$, ANOVA and Tukey's multiple comparison test) (Fig. 1A).

The volume of polyps detected along the entire intestinal tract of each mouse was calculated by considering polyps as hemispheres $\left(1 / 2 \times 3 / 4 \pi r^{3}\right)$. Analysis of the polyp volume revealed a statistically significant reduction in the OM-3R group as compared to animals fed with the standard diet for 5 weeks (ST1) and for 10 weeks (ST2) $(\mathrm{P}=0.03$ and $\mathrm{P}=0.002$, respectively, ANOVA and Tukey's multiple comparison test) (Fig. 1B). These results clearly indicated that n-3 PUFAs reversed the intestinal polyp formation in $\mathrm{Apc}^{\mathrm{Min} /+}$ mice.

Protein expression of estrogen receptors. To examine the underlying molecular mechanisms of polyp reversion, we studied the two cell signals of proliferation and of apoptosis.

The role of ER $\beta$ in counteracting tumor progression was confirmed. Fig. 2 shows a significant increase of ER $\beta$ protein expression in the OM-3R group versus the ST1 and ST2 groups $(\mathrm{P}=0.0002$ and $\mathrm{P}=0.0001$, respectively, ANOVA and Tukey's 

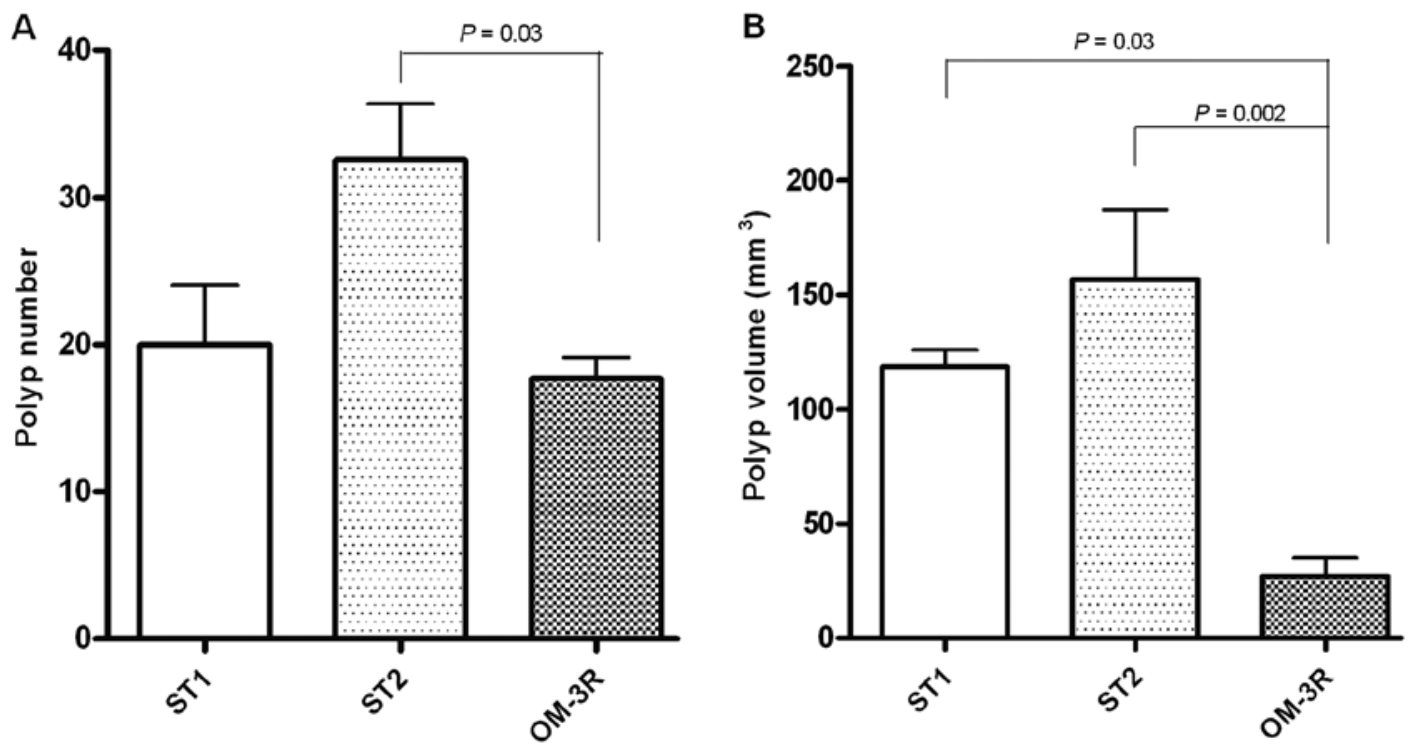

Figure 1. Total number and volume of intestinal polyps. (A) Number and (B) total volume of polyps in the whole intestinal tract from Apc ${ }^{\mathrm{Min} /+}$-treated groups: ST1, standard diet for 5 weeks; ST2, standard diet for 10 weeks; OM-3R, standard diet for 5 weeks plus n-3 polyunsaturated fatty acids (n-3 PUFAs)-enriched diet for another 5 weeks. Data are presented as the mean \pm SE. P $<0.05$ shows statistically significant differences (one-way analysis of variance and Tukey's multiple comparison test).
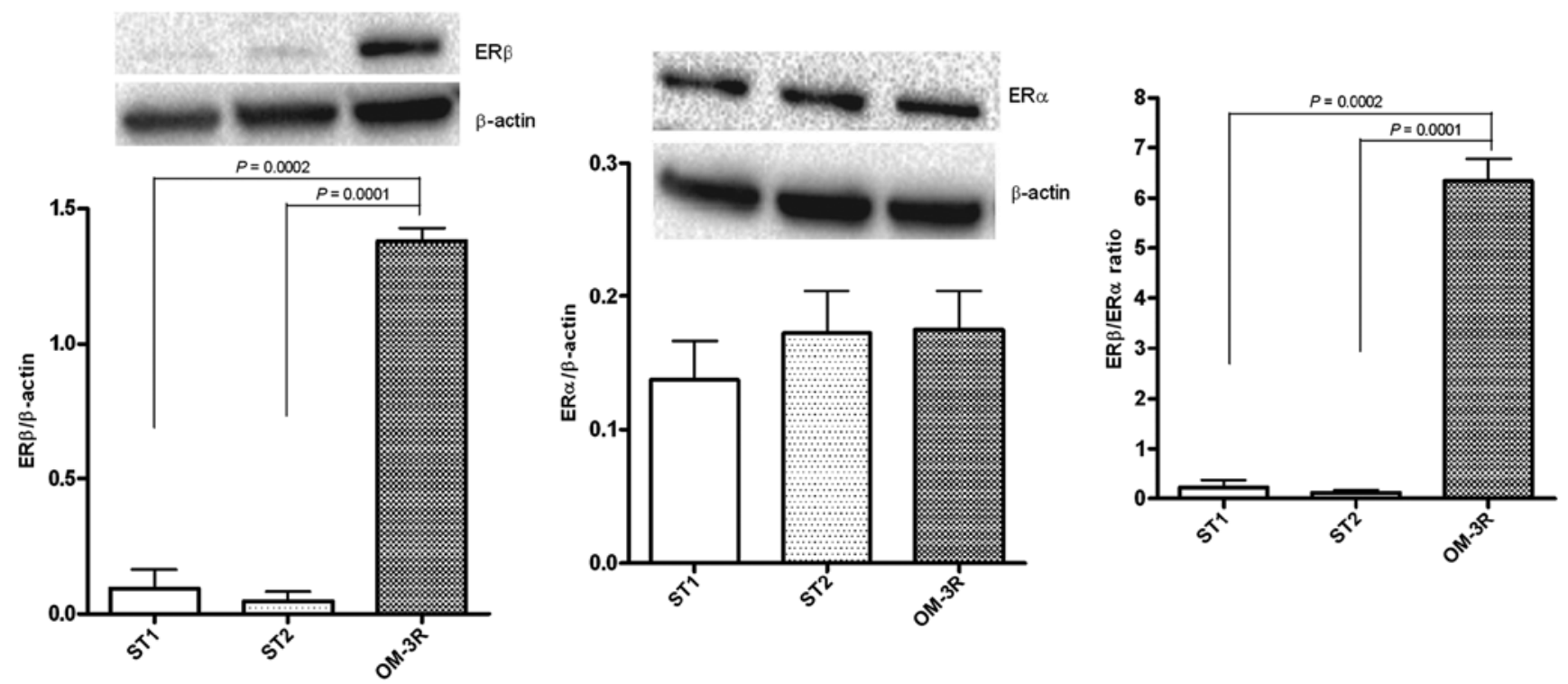

Figure 2. Protein expression of estrogen receptors. Western blot analysis of estrogen receptor levels in distal intestinal tissue from Apc ${ }^{\mathrm{Min} /}-$ treated groups. Graphics shows the normalized levels with $\beta$-actin of estrogen receptor $\beta$ (ER $\beta)$ and ER $\alpha$, as well as the levels of ER $\beta / E R \alpha$ ratio. ST1, standard diet for 5 weeks; ST2, standard diet for 10 weeks; OM-3R, standard diet for 5 weeks plus n-3 polyunsaturated fatty acids (n-3 PUFAs)-enriched diet for another 5 weeks. Data are presented as the mean \pm SE. $\mathrm{P}<0.05$ shows statistically significant differences (one-way analysis of variance and Tukey's multiple comparison test).

multiple comparison test), whereas the ER $\alpha$ protein levels were not altered. These results led to a significant induction of ER $\beta /$ $\mathrm{ER} \alpha$ ratio, evident in the OM-3R group as compared to the ST1 and ST2 groups $(\mathrm{P}=0.0002$ and $\mathrm{P}=0.0001$, respectively).

Protein expression of Shh and GLI-1. Since ER $\alpha$, a mediator of cell proliferation, regulates the Hh signaling pathway, we examined the protein expression levels of Shh and GLI-1 in mouse intestinal tissue. The results demonstrated that the two proteins were differentially expressed following the n-3 PUFAs diet treatment: Shh protein levels were significantly downregulated in the OM-3R group, whereas the GLI-1 protein expression was unmodified when compared to the ST1 and ST2 groups (Fig. 3).

Protein expression of STAT3Ser/STAT3 ratio and PIAS-3. To better define the molecular connection between n-3 PUFAs and cell proliferation, we evaluated the expression of p-STAT3, known to play a role in cell growth by upregulating the expression of anti-apoptotic genes. The results clearly demonstrated that p-STAT3Ser expression was significantly reduced in mice fed on n-3 PUFAs as compared to mice receiving only the standard diet for 5 weeks ( $\mathrm{P}=0.01$, ANOVA and Tukey's multiple comparison test) (Fig. 4A). Consequently, PIAS-3 
A
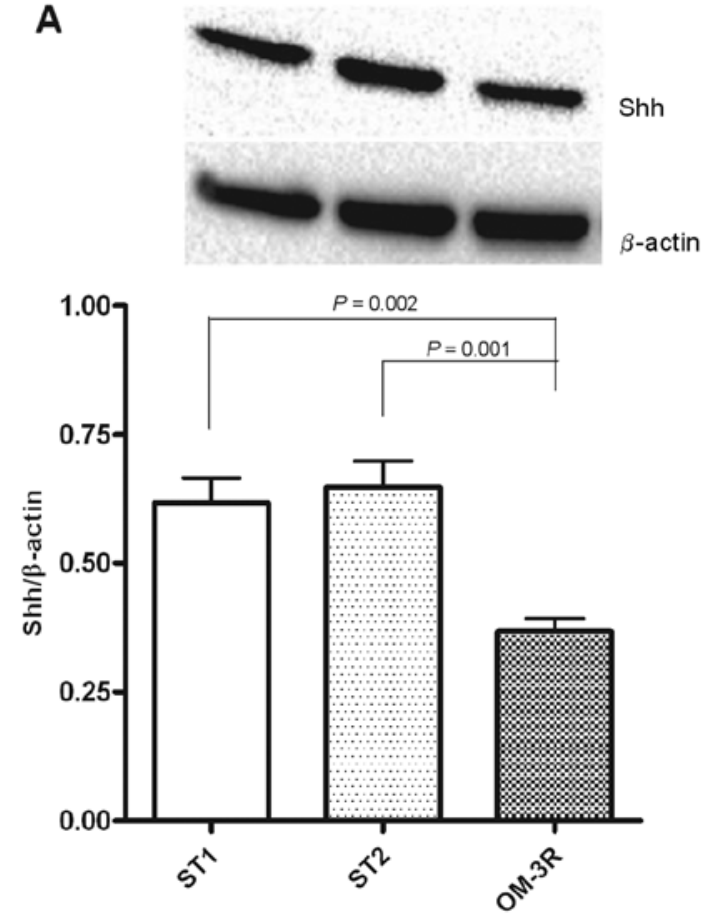

B
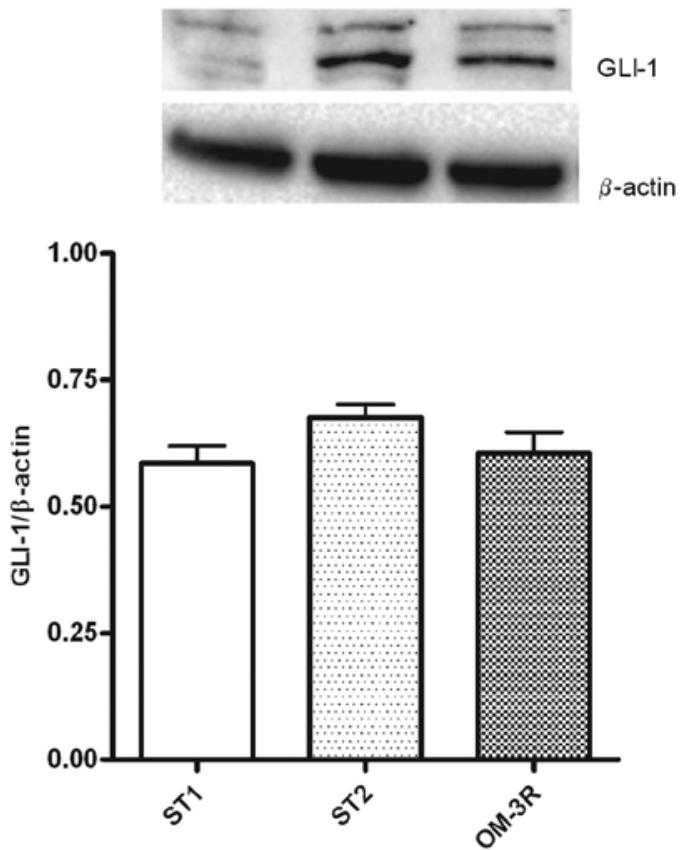

Figure 3. Protein expression of sonic hedgehog (Shh) and glioma-associated oncogene homolog-1 (GLI-1). Western blot analysis of (A) Shh and (B) GLI-1 levels in the distal intestinal tract from Apc ${ }^{\mathrm{Min} / \mathrm{t}}$-treated groups. ST1, standard diet for 5 weeks; ST2, standard diet for 10 weeks; OM-3R, standard diet for 5 weeks plus n-3 polyunsaturated fatty acids (n-3 PUFAs)-enriched diet for another 5 weeks. Data are presented as the mean \pm SE. $\mathrm{P}<0.05$ shows statistically significant differences (ANOVA and Tukey's multiple comparison test).

A
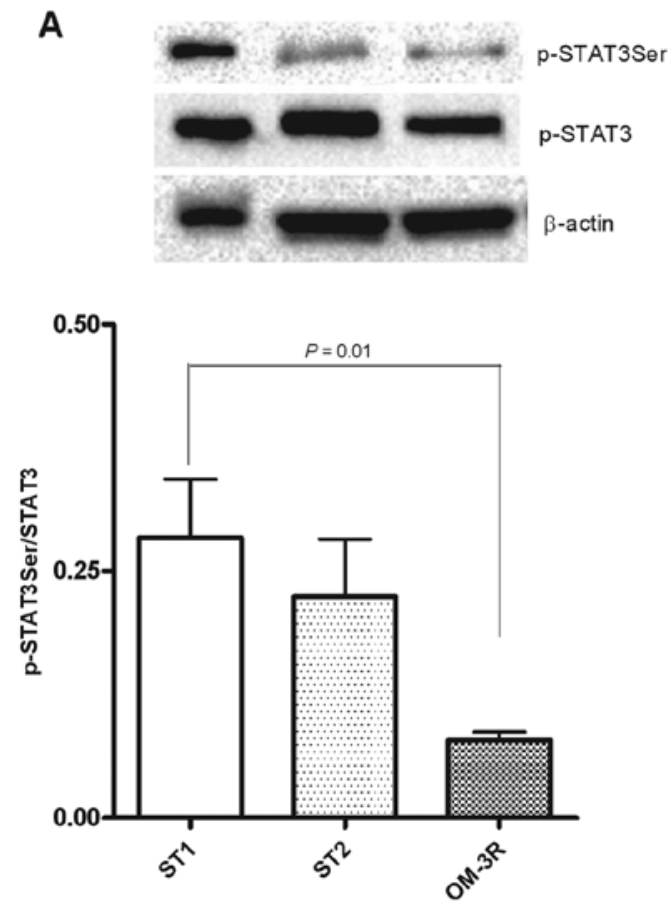

B
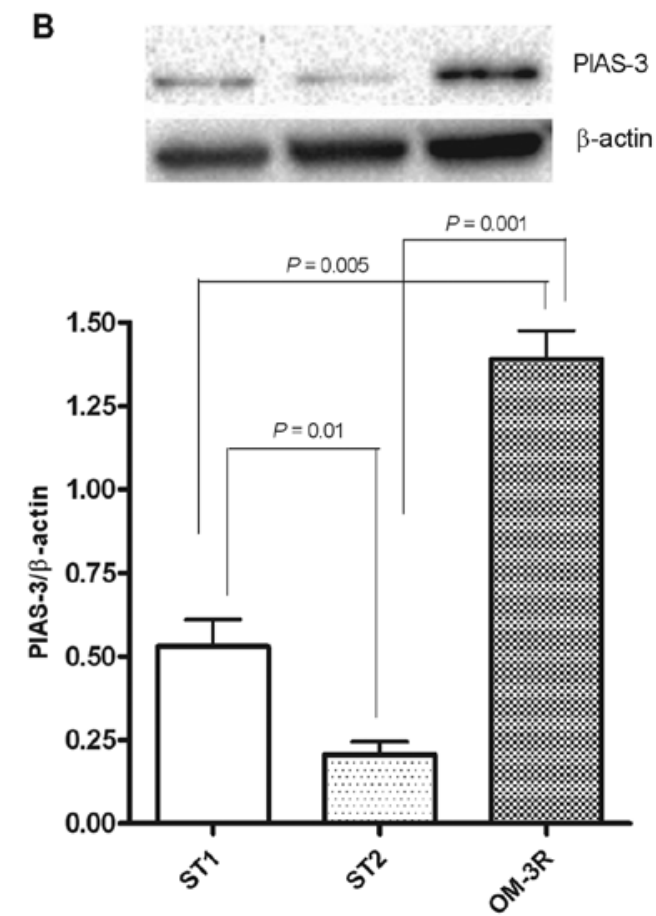

Figure 4. Protein expression of STAT3Ser/STAT3 ratio and PIAS-3. (A) Western blot analysis of the expression of the p-STAT3Ser/STAT3 ratio in distal intestinal tissue of $\mathrm{Apc}^{\mathrm{Min} /+}$ mice. (B) Western blot analysis of PIAS-3 protein in distal intestinal tissue of treated Apc ${ }^{\mathrm{Min} / \mathrm{+}}$ mice. ST1, standard diet for 5 weeks; ST2, standard diet for 10 weeks; OM-3R, standard diet for 5 weeks plus n-3 polyunsaturated fatty acids (n-3 PUFAs)-enriched diet for another 5 weeks. Data are presented as the mean $\pm \mathrm{SE}$. $\mathrm{P}<0.05$ shows statistically significant difference (one-way analysis of variance and Tukey's multiple comparison test).

levels, known to be an inhibitor of p-STAT3, were significantly increased in the OM-3R mouse group as compared to mice receiving only the standard diet, for 5 and 10 weeks $(\mathrm{P}=0.005$ and $\mathrm{P}=0.001$, respectively) (Fig. 4B). Fig. 4B shows that PIAS protein expression was highly downregulated after 10 weeks of treatment with the standard diet. 

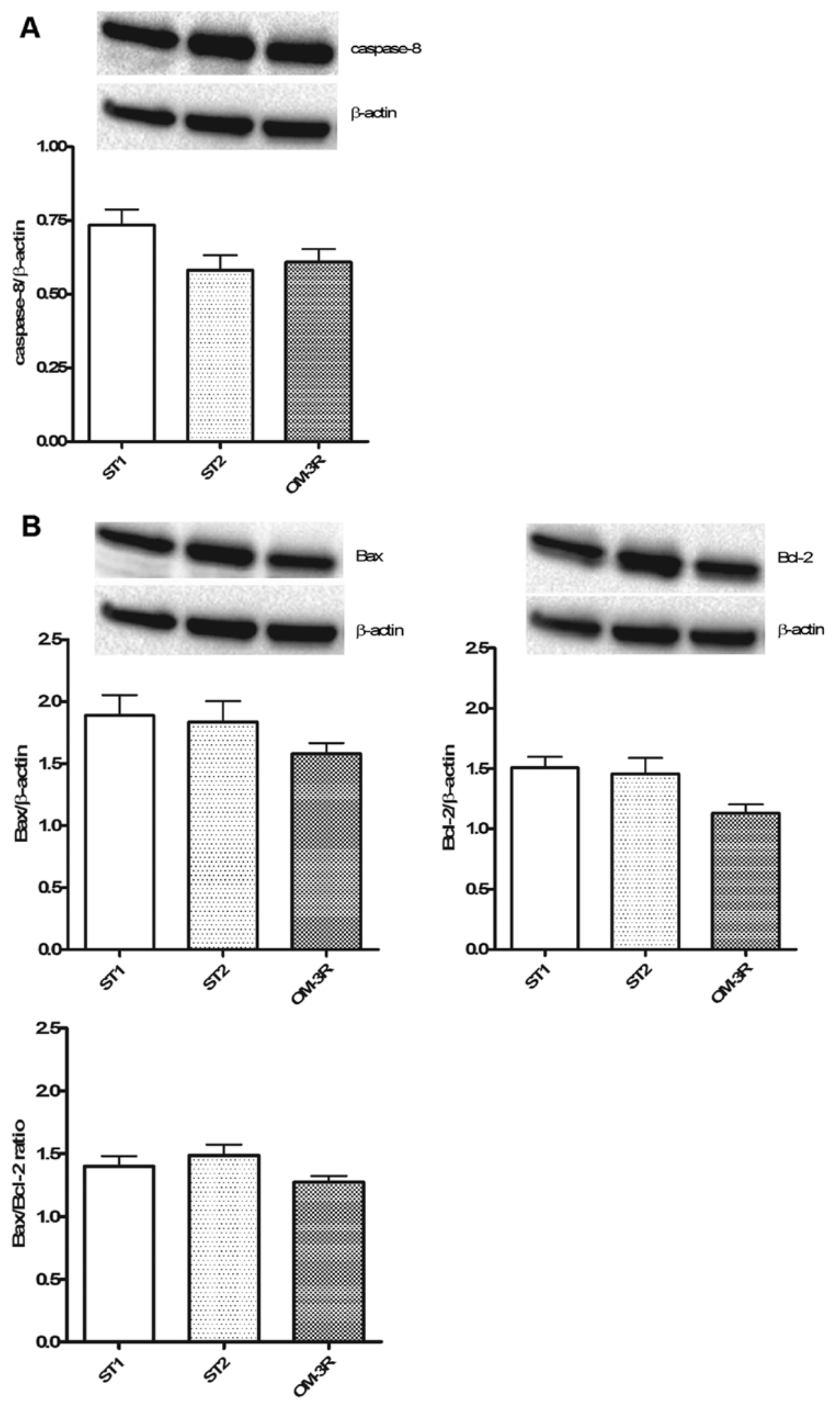

Figure 5. Expression level of apoptotic proteins. (A) Western blot analysis of caspase-8 protein expression in the distal intestinal tract from Apc ${ }^{\mathrm{Min} /+}$-treated groups. (B) Levels of Bax and Bcl-2 expression normalized with $\beta$-actin, as well as the levels of Bax/Bcl-2 ratio. ST1, standard diet for 5 weeks; ST2, standard diet for 10 weeks; OM-3R, standard diet for 5 weeks plus n-3 polyunsaturated fatty acids (n-3 PUFAs)-enriched diet for another 5 weeks. Data are presented as the mean $\pm \mathrm{SE}$. $\mathrm{P}<0.05$ shows statistically significant differences (ANOVA and Tukey's multiple comparison test).

Expression level of apoptotic proteins. The expression levels of caspase-8 (Fig. 5A), and Bax and Bcl-2 proteins (Fig. 5B) were determined. Apoptotic processes were not involved in the reversion process induced by $n-3$ PUFAs, i.e., no change in the caspase- 8 , Bax and $\mathrm{Bcl}-2$ protein expression was observed in the OM-3R group as compared to the ST1 and ST2 groups. 


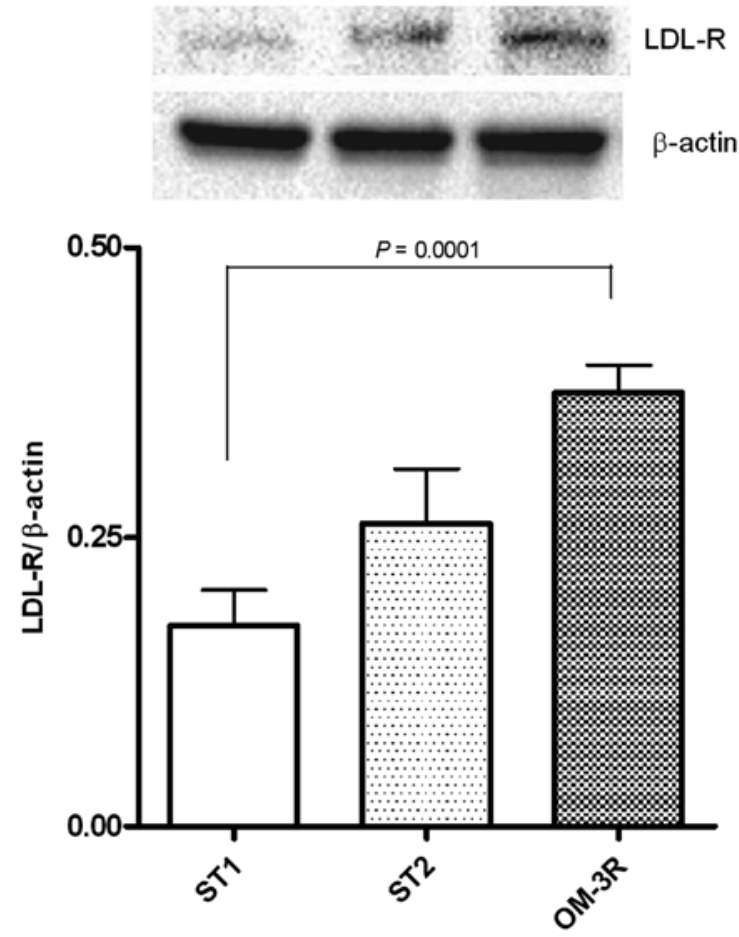

Figure 6. LDL-R protein expression. Western blot analysis of LDL-R protein in the distal intestinal tract from $\mathrm{Apc}^{\mathrm{Min} /+}$-treated groups. ST1, standard diet for 5 weeks; ST2, standard diet for 10 weeks; OM-3R, standard diet for 5 weeks plus n-3 polyunsaturated fatty acids ( $n-3$ PUFAs)-enriched diet for another 5 weeks. Data are presented as the mean $\pm \mathrm{SE}$. $\mathrm{P}<0.05$ shows statistically significant differences (ANOVA and Tukey's multiple comparison test).

$L D L-R$ protein expression and levels of FAS activity. The dietary n-3 PUFAs treatment, in intestinal mice tissue, caused a significant increase of LDL-R expression, able to exert an inhibitory effect on tumor cell growth (Fig. 6) as well as a strong reduction of FAS activity (Fig. 7). These results confirmed that natural compounds such as n-3 PUFAs can elicit their effects via the downregulation of lipogenic enzymes.

\section{Discussion}

The present study shows for the first time the ability of a diet enriched with n-3 PUFAs to invert the polyp formation process in a $\mathrm{Apc}^{\mathrm{Min} /+}$ mouse model.

Previous findings demonstrated in $\mathrm{Apc}^{\mathrm{Min} /+}$ mice that n-3 PUFAs significantly reduced the number and volume of polyps, through a decrease of cell proliferation and an increase of apoptosis in the adenomatous tissue (9). By contrast, the reverse process of polyp development, is likely due to the activation of anti-proliferative mechanisms that exclude cell apoptotic processes.

n-3 PUFAs were able to suppress intestinal polyps in $\mathrm{Apc}^{\mathrm{Min} /+}$ mice and significantly reverse polyp development associated with the downregulation of cell proliferation markers and with the induction of ER $\beta$ and LDL-R, which are negative modulators of cell proliferation.

p-STAT3 has been shown to have pro-proliferative effects and is responsible for the activation of metabolic pathways involved in the regulation of cell proliferation (25-28). Its reduction, following n-3 PUFAs treatment, is an index of the shutdown of cell proliferation in mouse intestinal tissue.

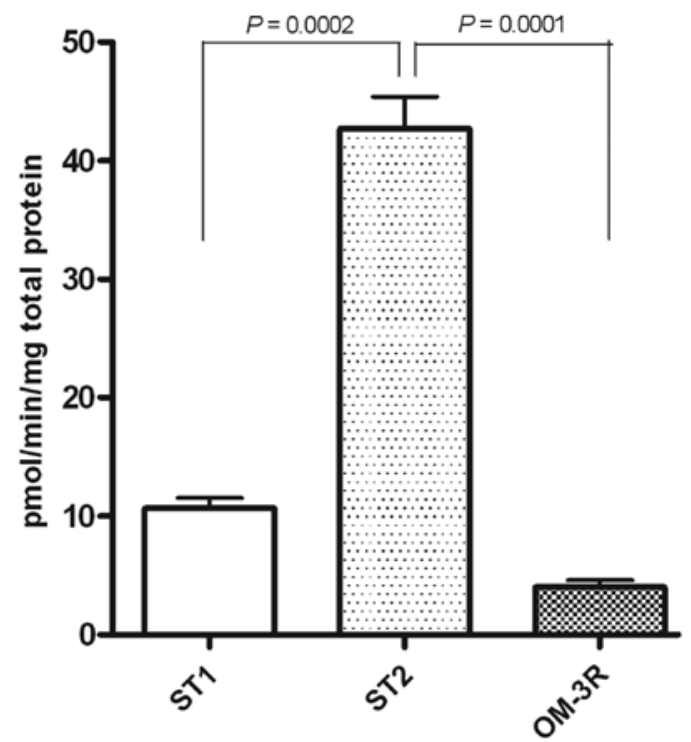

Figure 7. Levels of fatty acid synthase (FAS) activity. FAS enzymatic activity in the distal intestinal tract from $\mathrm{Apc}^{\mathrm{Min} /+}$-treated groups. ST1, standard diet for 5 weeks; ST2, standard diet for 10 weeks; OM-3R, standard diet for 5 weeks plus n-3 polyunsaturated fatty acids (n-3 PUFAs)-enriched diet for another 5 weeks. Data are presented as the mean \pm SE. $\mathrm{P}<0.05$ shows statistically significant differences (ANOVA and Tukey's multiple comparison test).

Consequently, PIAS-3 levels were induced, since PIAS-3 is known to control the extent and the duration of STAT3 activity in cells preventing its oncogenic function (29).

Several chemopreventive agents, such as olive oil, n-3 PUFAs, curcumin and silymarin $(9,11,30)$ have been demonstrated to suppress the spontaneous formation of intestinal tumors in the $\mathrm{Apc}^{\mathrm{Min} /+}$ mouse, confirming a key role of diet in modulating colon cancer risk.

Different mechanisms have been suggested to explain the protective effect of n-3 PUFAs on colon cancer development. Authors have suggested that their antineoplastic effects would involve the incorporation of n-3 PUFAs into cellular membranes replacing the n-6 PUFAs with a consequent reduction of inflammation $(3,6)$.

Previously, we demonstrated that the anti-proliferative effects of dietary n-3 PUFAs were associated with an inhibition of FAS and HMGCoAR gene expression and activity and to an increased $\mathrm{ER} \beta / \mathrm{ER} \alpha$ ratio (9).

In the present study, we showed that induction of the ER $\beta / E R \alpha$ ratio is also involved in the reverse process of polyp development associated with the upregulation of LDL-R expression.

LDL-R has been found to play a role in cell growth and tumorigenesis (31). Previously, we showed that LDL-R was little expressed in colon cancer and that the absence of LDL-R predicted shorter survival in CRC patients (32). Thus, the factors that upregulate LDL-R expression in normal and tumor cells, consequently, are able to control cell proliferation and transformation.

In addition, dietary n-3 PUFAs exerted a decrease of p-STAT3Ser protein expression and FAS activity, confirming the central role of n-3 PUFAs in the regulation of cell proliferation metabolic pathways, as previously demonstrated.

In conclusion, n-3 PUFAs-induced metabolic changes are able to counteract intestinal polyp formation in the mice and to 
revert polyp growth. This noteworthy finding is important for a translational study evaluating the therapeutic role of dietary components on human health. In particular, the marked effect of diets on polyp development in the absence of toxicity makes n-3 PUFAs an excellent candidate for the prevention and treatment of subjects with gastrointestinal diseases.

\section{References}

1. Caygill CP, Charlett A and Hill MJ: Fat, fish, fish oil and cancer. Br J Cancer 74: 159-164, 1996.

2. Kato I, Akhmedkhanov A, Koenig K, Toniolo PG, Shore RE and Riboli E: Prospective study of diet and female colorectal cancer: The New York University Women's Health Study. Nutr Cancer 28: 276-281, 1997.

3. Dupertuis YM, Meguid MM and Pichard C: Colon cancer therapy: New perspectives of nutritional manipulations using polyunsaturated fatty acids. Curr Opin Clin Nutr Metab Care 10: 427-432, 2007

4. Chapkin RS, Seo J, McMurray DN and Lupton JR: Mechanisms by which docosahexaenoic acid and related fatty acids reduce colon cancer risk and inflammatory disorders of the intestine. Chem Phys Lipids 153: 14-23, 2008.

5. Petrik MB, McEntee MF, Chiu CH and Whelan J: Antagonism of arachidonic acid is linked to the antitumorigenic effect of dietary eicosapentaenoic acid in Apc(Min/+) mice. J Nutr 130: $1153-1158,2000$.

6. Fini L, Piazzi G, Ceccarelli C, Daoud Y, Belluzzi A, Munarini A, Graziani G, Fogliano V, Selgrad M, Garcia M, et al: Highly purified eicosapentaenoic acid as free fatty acids strongly suppresses polyps in Apc(Min/+) mice. Clin Cancer Res 16: 5703-5711, 2010

7. West NJ, Clark SK, Phillips RK, Hutchinson JM, Leicester RJ, Belluzzi A and Hull MA: Eicosapentaenoic acid reduces rectal polyp number and size in familial adenomatous polyposis. Gut 59: 918-925, 2010.

8. Notarnicola M, Messa C, Refolo MG, Tutino V, Miccolis A and Caruso MG: Polyunsaturated fatty acids reduce fatty acid synthase and hydroxy-methyl-glutaryl CoA-reductase gene expression and promote apoptosis in HepG2 cell line. Lipids Health Dis 10: 10, 2011.

9. Barone M, Notarnicola M, Caruso MG, Scavo MP, Viggiani MT, Tutino V, Polimeno L, Pesetti B, Di Leo A and Francavilla A: Olive oil and omega-3 polyunsaturated fatty acids suppress intestinal polyp growth by modulating the apoptotic process in $\mathrm{Apc}^{\mathrm{Min} /+}$ mice. Carcinogenesis 35: 1613-1619, 2014.

10. Di Leo A, Barone M, Maiorano E, Tanzi S, Piscitelli D, Marangi S, Lofano K, Ierardi E, Principi M and Francavilla A: ER-beta expression in large bowel adenomas: Implications in colon carcinogenesis. Dig Liver Dis 40: 260-266, 2008.

11. Barone M, Tanzi S, Lofano K, Scavo MP, Pricci M, Demarinis L, Papagni S, Guido R, Maiorano E, Ingravallo G, et al: Dietary-induced ERbeta upregulation counteracts intestinal neoplasia development in intact male $\mathrm{Apc}^{\mathrm{Min} /+}$ mice. Carcinogenesis 31: 269-274, 2010.

12. Barone M, Scavo MP, Papagni S, Piscitelli D, Guido R, Di Lena M, Comelli MC and Di Leo A: ER $\beta$ expression in normal, adenomatous and carcinomatous tissues of patients with familial adenomatous polyposis. Scand J Gastroenterol 45: $1320-1328,2010$

13. Konstantinopoulos PA, Kominea A, Vandoros G, Sykiotis GP, Andricopoulos P, Varakis I, Sotiropoulou-Bonikou G and Papavassiliou AG: Oestrogen receptor beta (ERbeta) is abundantly expressed in normal colonic mucosa, but declines in colon adenocarcinoma paralleling the tumour's dedifferentiation. Eur J Cancer 39: 1251-1258, 2003.

14. Rudolph A, Toth C, Hoffmeister M, Roth W, Herpel E, Jansen L, Marx A, Brenner $\mathrm{H}$ and Chang-Claude J: Expression of oestrogen receptor $\beta$ and prognosis of colorectal cancer. Br J Cancer 107: 831-839, 2012.
15. Weihua Z, Andersson S, Cheng G, Simpson ER, Warner M and Gustafsson JA: Update on estrogen signaling. FEBS Lett 546: 17-24, 2003.

16. Thomas C and Gustafsson JÅ: The different roles of ER subtypes in cancer biology and therapy. Nat Rev Cancer 11: 597-608, 2011.

17. Kameda C, Nakamura M, Tanaka H, Yamasaki A, Kubo M, Tanaka M, Onishi $\mathrm{H}$ and Katano M: Oestrogen receptor- $\alpha$ contributes to the regulation of the hedgehog signalling pathway in ERalpha-positive gastric cancer. Br J Cancer 102: 738-747, 2010.

18. Wang H, Li YY, Wu YY and Nie YQ: Expression and clinical significance of hedgehog signaling pathway related components in colorectal cancer. Asian Pac J Cancer Prev 13: 2319-2324, 2012.

19. Bian YH, Huang SH, Yang L, Ma XL, Xie JW and Zhang HW: Sonic hedgehog-Gli1 pathway in colorectal adenocarcinomas. World J Gastroenterol 13: 1659-1665, 2007.

20. Gulino A, Ferretti E and De Smaele E: Hedgehog signalling in colon cancer and stem cells. EMBO Mol Med 1: 300-302, 2009.

21. Notarnicola M, Messa C, Pricci M, Guerra V, Altomare DF, Montemurro S and Caruso MG: Up-regulation of 3-hydroxy-3methylglutaryl coenzyme A reductase activity in left-sided human colon cancer. Anticancer Res 24: 3837-3842, 2004.

22. Caruso MG and Notarnicola M: Biochemical changes of mevalonate pathway in human colorectal cancer. Anticancer Res 25: 3393-3397, 2005

23. Swinnen JV, Brusselmans K and Verhoeven G: Increased lipogenesis in cancer cells: New players, novel targets. Curr Opin Clin Nutr Metab Care 9: 358-365, 2006.

24. Notarnicola M, Miccolis A, Tutino V, Lorusso D and Caruso MG: Low levels of lipogenic enzymes in peritumoral adipose tissue of colorectal cancer patients. Lipids 47: 59-63, 2012.

25. Goodman WA, Young AB, McCormick TS, Cooper KD and Levine AD: Stat 3 phosphorylation mediates resistance of primary human $\mathrm{T}$ cells to regulatory $\mathrm{T}$ cell suppression. J Immunol 186: 3336-3345, 2011.

26. Gao SP, Mark KG, Leslie K, Pao W, Motoi N, Gerald WL, Travis WD, Bornmann W, Veach D, Clarkson B, et al: Mutations in the EGFR kinase domain mediate STAT3 activation via IL-6 production in human lung adenocarcinomas. J Clin Invest 117: 3846-3856, 2007.

27. Koukos G, Polytarchou C, Kaplan JL, Morley-Fletcher A, Gras-Miralles B, Kokkotou E, Baril-Dore M, Pothoulakis C, Winter HS and Iliopoulos D: MicroRNA-124 regulates STAT3 expression and is down-regulated in colon tissues of pediatric patients with ulcerative colitis. Gastroenterology 145: 842-52.e2, 2013.

28. Behera R, Kumar V, Lohite K, Karnik S and Kundu GC: Activation of JAK2/STAT3 signaling by osteopontin promotes tumor growth in human breast cancer cells. Carcinogenesis 31: 192-200, 2010.

29. Borghouts C, Tittmann H, Delis N, Kirchenbauer M, Brill B and Groner B: The intracellular delivery of a recombinant peptide derived from the acidic domain of PIAS3 inhibits STAT3 transactivation and induces tumor cell death. Mol Cancer Res 8: 539-553, 2010.

30. Pettan-Brewer C, Morton J, Mangalindan R and Ladiges W: Curcumin suppresses intestinal polyps in APC Min mice fed a high fat diet. Pathobiol Aging Age Relat Dis 1: 7013, 2011.

31. Notarnicola M, Messa C, Refolo MG, Tutino V, Miccolis A and Caruso MG: Synergic effect of eicosapentaenoic acid and lovastatin on gene expression of HMGCoA reductase and LDL receptor in cultured HepG2 cells. Lipids Health Dis 30: 135,2010

32. Caruso MG, Notarnicola M, Santilo MR, Cavallini A, Di Leo A: Enhanced 3-hydroxy-3-methyl-glutaryl coenzyme a reductase activity in human colorectal cancer not expressing low density lipoprotein receptor. Anticancer Res 19: 451-454, 1999. 